\title{
Comparative collisionless alpha particle confinement in stellarator reactors with the XGC gyrokinetic code
}

\author{
M. D. J. Cole, ${ }^{1, \text { a) }}$ R. Hager, ${ }^{1}$ T. Moritaka, ${ }^{2}$ S. Lazerson, ${ }^{1}$ R. Kleiber, ${ }^{3}$ S. Ku, ${ }^{1}$ and C. S. Chang $^{1}$ \\ 1) Princeton Plasma Physics Laboratory, \\ Princeton University, Princeton, New Jersey 08543, USA \\ ${ }^{2)}$ National Institute for Fusion Science, \\ Toki 509-5292, Japan \\ 3) Max Planck Institute for Plasma Physics, \\ D-17491 Greifswald, Germany
}

(Dated: 1 February 2019)

Whole volume simulations of stellarators are necessary to address a number of important physics and engineering issues, including turbulent transport prediction and optimising divertor operation. In this work, advances in development of a whole volume stellarator capability for the global gyrokinetic particle-in-cell code XGC are detailed. A 3D interpolation of the equilibrium magnetic field to the last close flux surface, calculated using the VMEC ideal magnetohydrodynamic equilibrum code, has been implemented. Orbit tracing with the XGC code is verified for the Wendelstein 7-X stellarator by comparison with the BEAMS3D and EUTERPE codes. The XGC stellarator version has then been used to consider collisionless alpha particle confinement in representative quasi-isodynamic and quasi-axisymmetric designs. It has been shown that, at least without further optimisation for energetic particle confinement, the Wendelstein 7-X-like quasi-isodynamic reactor design exhibits better alpha particle confinement.

\section{INTRODUCTION}

Stellarators are toroidal magnetic confinement fusion devices in which finite rotational transform is generated primarily by shaping of the magnetic field coils rather than a toroidal plasma current. In order to produce a rotational transform, the magnetic field coils cannot generate an entirely axisymmetric field ${ }^{1}$. Stellarators are therefore intrinsically 3D magnetic systems. Interest in stellarators as commercially exploitable devices for power generation arises mostly from the benefits of eliminating plasma instabilities driven by a radial plasma current gradient, such as disruptions, and recirculating power losses involved in driving current.

To model stellarators effectively, adequate information about the 3D geometry and its global effect on plasma behaviour must be considered. Unlike in an axisymmetric device, the magnetic geometry varies between flux tubes on the same surface. It has been shown that flux tube simulations of core microturbulence are therefore insufficient, and a flux surface global approach is necessary $^{2}$. Furthermore, it has been shown that neoclassical transport and microturbulence undergo multiscale interactions in toroidal magnetic fusion devices generally $^{3-5}$. Since microturbulent transport should dominate in at least the edge and outer core of an optimised stellarator, while neoclassical transport should continue to dominate in the inner core ${ }^{6}$, this is likely to be especially so in optimised stellarators and a radially global model is also likely needed.

Few gyrokinetic codes are able to consider stellarator geometries globally in both radius and flux surface. One

\footnotetext{
a) mcole@pppl.gov
}

such code is EUTERPE, a delta-f gyrokinetic ParticleIn-Cell (PIC) code, which models the plasma volume to the last closed flux surface ${ }^{7}$. It has been used to perform simulations of neoclassical transport ${ }^{8-10}$ and linear microinstabilities underlying microturbulence in stellarators $^{11-13}$. This code and the GTC code have been applied to electromagnetic Alfvénic physics in stellarators with hybrid models ${ }^{14,15}$, and recently the GT5D code has been extended and used to model neoclassical transport in the Large Helical Device ${ }^{16}$. However, no whole volume code capable of modelling kinetic physics in stellarators currently exists.

On-going developments to XGC (X-point Gyrokinetic Code) are aimed at filling this gap. XGC is a whole volume total-f gyrokinetic PIC $\operatorname{code}^{17-19}$, which has the unusual capability for neutral particle recycling in diverted geometry. XGC has so far been applied to tokamaks including the edge $\mathrm{e}^{20,21}$. In this work, these developments and their application to stellarator physics are detailed. So far, XGC has been extended to include a model of the $3 \mathrm{D}$ equilibrium magnetic field of a stellarator to the last closed flux surface. This field is calculated initially with the VMEC ideal MHD equilibrium $\operatorname{code}^{22}$, which can treat general stellarator geometry assuming nested flux surfaces.

One important aspect of stellarator physics can already be treated with this model, confinement of energetic particles. Historically, axisymmetric tokamaks have been favoured over non-axisymmetric stellarators for fusion applications due to poorer particle and energy confinement in non-axisymmetric toroidal fields. In general, trapped particle orbits are not confined in stellarators. In recent decades, considerable effort has been made to design non-axisymmetric fields for maximum particle confinement. Tokamaks themselves increasingly also take 
advantage of non-axisymmetric fields to enhance stability and control power and particle exhaust ${ }^{23,24}$. At the same time, analytical and numerical tools have been applied to the optimisation of particle transport in stellarators.

One approach is to adopt 'quasi-symmetries', whereby a (possibly non-axial) approximate symmetry exists in the particle orbits in a toroidally inhomogenous field. Another approach is to optimise a non-symmetric configuration to minimise the time averaged radial drift of particles from a flux surface ${ }^{11,25}$. Both approaches require numerical analysis to find a desireable magnetic configuration that can be generated by buildable coils, and a broad space of possible designs exists. Although optimisation schemes, such as quasi-isodynamicity, greatly reduce the proportion of unconfined orbits, they are imperfect. Furthermore, for existing designs optimisation has been carried out for thermal particles, and it is unclear to what extent this optimisation carries over to fast particle orbits. A loss mechanism called stochastic diffusion, present in stellarators, also means that ordinarily confined orbits can result in losses even without collisions $^{26,27}$. If these loss mechanisms are not sufficiently suppressed, alpha particle losses could result in damage to the first wall of the device or failure of the fusion reaction to be self-sustaining. For a reactor to be commercially viable, energetic alpha particles should be well enough confined for the plasma to be self-heating.

In this article, the numerical model for stellarators so far implemented in XGC will be detailed and verification checks performed with a W7-X equilibrium in Section II. This analysis will be extended to a comparison of a W7-X-like (quasi-isodynamic) and NCSX-like (quasiaxisymmetric) reactor in Section III, and collisionless loss calculations detailed. This comparison allows a baseline quantitative assessment of the relative quality of current reactor designs for this important stellarator confinement property. The article concludes in Section IV.

\section{MODEL VERIFICATION}

$\mathrm{XGC}$ is a total-f gyrokinetic PIC code. In this paper, however, the fluctuating fields will not be considered. Marker particles are pushed according to the guiding centre equations of motion ${ }^{19}$,

$$
\begin{array}{r}
\dot{\vec{R}}_{s}=\frac{1}{D}\left[v_{\|} \vec{b}+\frac{m_{s} v_{\|}^{2}}{q_{s}} \frac{\nabla B \times \vec{b}}{B}+\frac{1}{q_{s} B} \vec{b} \times \mu \nabla B\right] \\
\dot{v}_{\| s}=-\frac{\mu}{m_{s} D}\left(\vec{b}+\frac{m_{s} v_{\|}}{q_{s}} \nabla B \times \vec{b}\right) \cdot \nabla B
\end{array}
$$

where

$$
D=1+v_{\|} \frac{\vec{b} \cdot(\nabla \times \vec{b})}{B},
$$

$q_{s}$ is the species charge, $m_{s}$ the species mass, $\vec{B}$ the background magnetic field, $\vec{B}=B \vec{b}$, and $\mu$ is the magnetic moment.
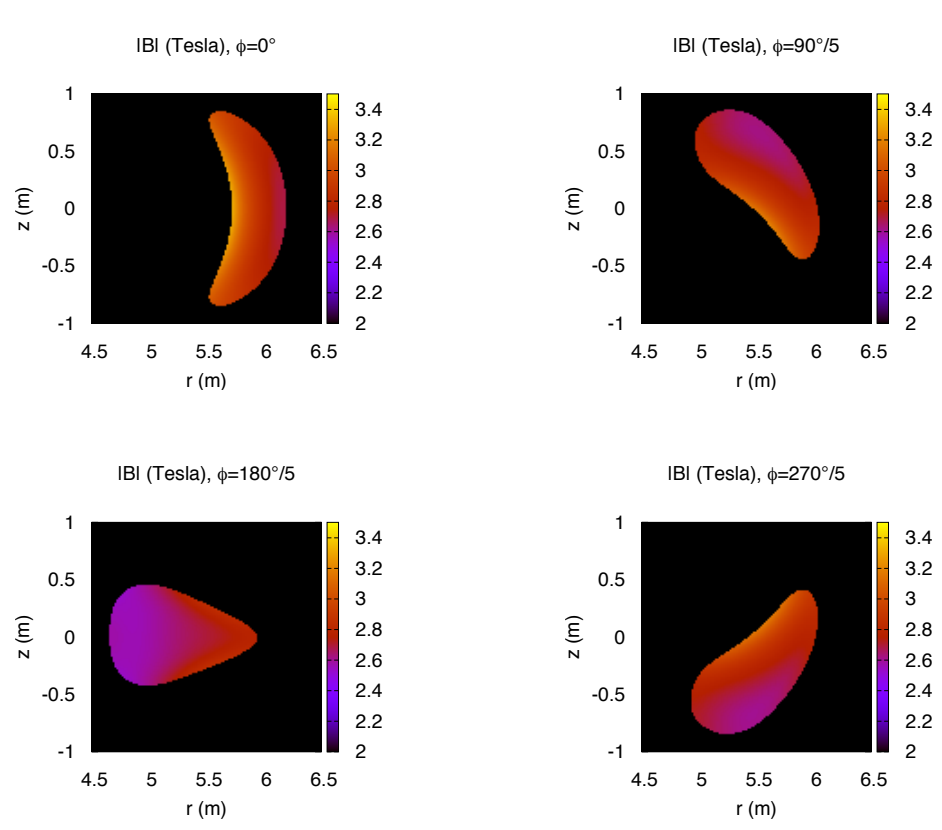

FIG. 1: The equilibrium magnetic field, $\vec{B}$, in $R$ and $Z$ at four different values of $\phi$ in the five-fold periodic stellarator Wendelstein 7-X.

Markers are initialised with given initial position, energy and pitch angle. The background magnetic field, $\vec{B}$, is calculated using the VMEC code with a fixed boundary, mapped to cylindrical coordinates and interpolated for the 3D domain used by XGC. The W7$\mathrm{X}$ equilibrium profiles chosen are a pressure profile of $P(s)=P_{0}\left(1-2 s^{2}+s^{4}\right)$ with a plasma beta on axis of $3 \%$, and an iota profile as in the standard configuration $^{28-30}$. A toroidal current is applied so as to produce an iota profile as in the vacuum case. Stellarators are generally periodic devices, so $\vec{B}$ is calculated only for a single field period. In figure 1 , the magnetic field strength at four different toroidal locations in a W7-X field period is depicted.

The BEAMS3D code was written to model Neutral Beam Injection (NBI) in stellarators ${ }^{31}$. It solves equations similar to eq. 1 and eq. 2 in a stellarator equilibrium,

$$
\begin{array}{r}
\dot{\vec{R}}_{s}=\frac{\vec{b}}{q_{s} B} \times\left(\mu \nabla B+\frac{m_{s} v_{\|}^{2}}{B}(\vec{b} \cdot \nabla) B\right)+v_{\|} \vec{b} \\
\dot{v}_{\| s}=-\frac{\mu}{m_{s}} \vec{b} \cdot(\nabla B) .
\end{array}
$$

BEAMS3D has been run for W7-X, and therefore provides an effective benchmark for stellarators with XGC. In figure $2 \mathrm{a}$, a single particle is traced with the BEAMS3D code and XGC. In this and all subsequent verification benchmarks, protium ions are considered. The evolution of its position in one dimension in Cartesian coordinates is plotted as a line (for BEAMS3D) and 
points (for XGC). One can see that, even at times very long compared to typical turbulence simulations, the two codes remain in close agreement.

The stellarator version of XGC can also be benchmarked with the EUTERPE code. EUTERPE is a gyrokinetic delta-f PIC code which models stellarator physics to the last closed flux surface ${ }^{7}$. It solves equivalent equations for the unperturbed particle motion in cylindrical coordinates. It has been applied to study a number of important stellarator phenomena, including neoclassical transport, linear ion-scale instabilities, and Alfvénic physics. The EUTERPE code has been adapted to permit individual particle orbit tracing.

For a more detailed test we can compare the loss fraction of particles initialised on a given flux surface $\left(s=\psi / \psi_{L C S F}=0.015625\right)$ with varying energy, using all three codes. Particles are initialised with a range of energies between $25 \mathrm{keV}$ and $200 \mathrm{keV}$, in increments of $25 \mathrm{keV}$. At each energy, 4096 particles are initialised, distributed uniformly in poloidal angle at 16, and toroidal angle (within one field period) at 8 , different positions. At each location particles are then distributed uniformly in pitch angle, $\theta_{p}=\tan ^{-1}\left(v_{\perp} / v_{\|}\right)$, between $\theta_{p}=61.8^{\circ}$ and $\theta_{p}=89.1^{\circ}$, at 16 different values, for both positive and negative initial parallel velocities. Both codes use the last closed flux surface as the plasma boundary. Particles are followed to $t=0.05 \mathrm{~s}$, at which point the majority of physical particles would have been thermalised by collisions.

In figure $2 \mathrm{~b}$, the proportion of particles lost is plotted. Good qualitative agreement is seen between the three codes for the loss fraction across the range of energies. Exact agreement is not expected, because the loss mechanism is stochastic. Small differences in the numerical implementation are therefore able to more significantly influence the final result.

The EUTERPE code can also be used to benchmark $\mathrm{XGC}$ for energetic particle loss fraction across the full radius of the device to the last closed flux surface. In figure 3 , the loss fraction for $100 \mathrm{keV}$ ions distributed as above but now born at different flux surfaces, designated by the flux label $s$, is plotted. Good agreement is seen between the three codes over the whole volume. By comparison between the three codes, it was discovered the need for an accurate extrapolation scheme to ensure that spurious numerical ripple is not introduced near the edge region. This was necessary to ensure accurate results for energetic particles born near the last closed flux surface.

In all these simulations, and as is clear from inspection of the equations, electrostatic and magnetic field perturbations arising due to the particle motion are neglected. The VMEC code also does not permit the calculation of magnetic islands, whose presence may impact the loss rates for fast particles. Furthermore, finite Larmor radius effects are neglected. These approximations are consistent between all the particle-following codes.

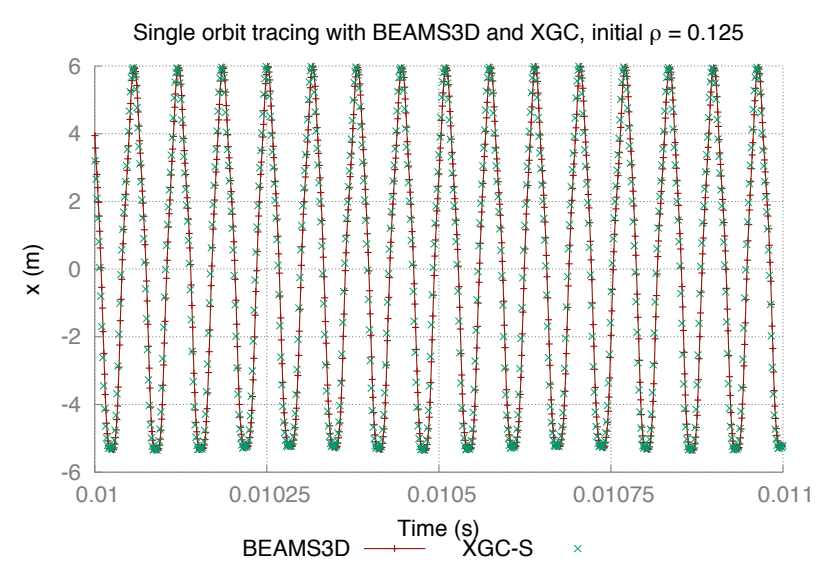

(a) Evolution of the position of a particle in $x$ in a Cartesian coordinate system.

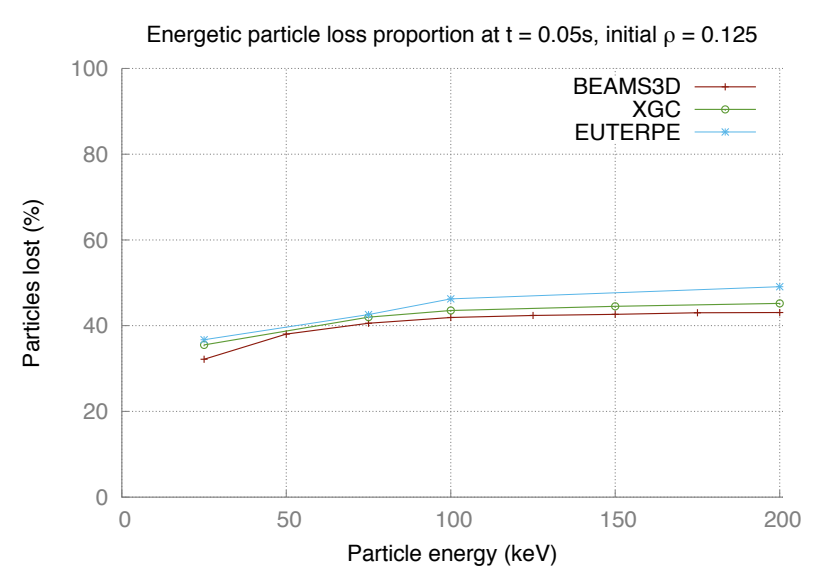

(b) Loss fraction of particles for a range of birth energies.

FIG. 2: Comparisons between the XGC, EUTERPE and BEAMS3D codes in Wendelstein 7-X geometry.

\section{COLLISIONLESS ALPHA PARTICLE CONFINEMENT IN QUASI-ISODYNAMIC AND QUASI-AXISYMMETRIC STELLARATOR REACTORS}

In tokamaks, the magnetic ripple is known to result in collisionless loss by a process called stochastic diffu$\operatorname{sion}^{26,27,32}$. This occurs because particles can be displaced radially as they move from regions where they are locally passing to locally trapped. In stellarators, which usually have much larger effective ripple, this effect is much more pronounced. Loss of energetic particles can therefore be prohibitive even without considering collisional loss mechanisms.

Since alpha particle loss rates are a decisive charac- 


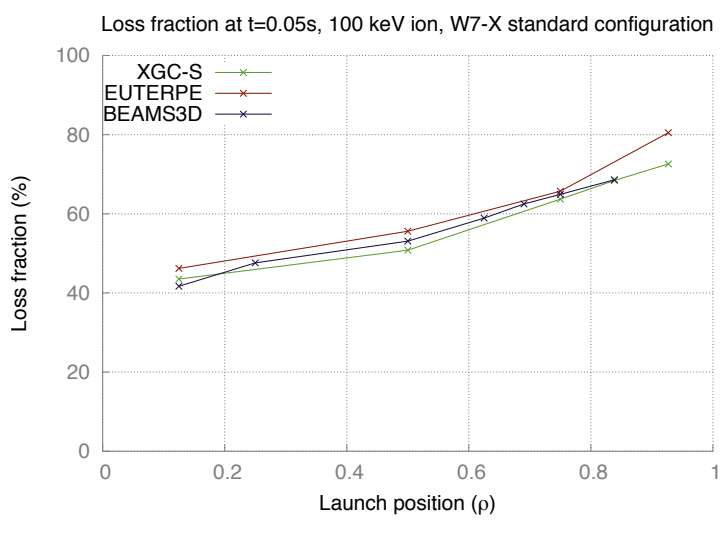

FIG. 3: A comparison of the loss fraction of $100 \mathrm{keV}$ ions born on a range of initial surfaces calculated by XGC and EUTERPE.

teristic of any possible stellarator design, it is interesting to compare the performance of different designs. In this work, two designs are compared. One is a quasiisodynamic, 'Wendelstein 7-X-like', reactor design, based on a $3.7 \times$ scaled up Wendelstein 7 -X configuration with a stronger $4.66 \mathrm{~T}$ magnetic field on-axis. Average major radius is $22 \mathrm{~m}$ and minor radius $1.8 \mathrm{~m}$. The normalised plasma pressure $\beta$ on-axis is $4.3 \%$, and the pressure profile is of the form $P(s)=P_{0}(1-s)$. The other is derived from the ARIES-CS ${ }^{33}$ reactor study, a quasiaxisymmetric designe based on the National Compact Stellarator Experiment. It has a $\beta$ and magnetic field on-axis of $4 \%$ and $5.63 \mathrm{~T}$ respectively, and a projected experimental-like pressure profile. Average major radius is $9.3 \mathrm{~m}$ and minor radius $1.6 \mathrm{~m}$. The rotational transform profiles of the two devices are compared in figure 5 .

The quasi-isodynamic design can be viewed as 'quasipoloidal', confining most trapped particles to precess around a given field period. The quasi-axisymmetric design, meanwhile, resembles a tokamak but where reduced plasma current is achieved at the cost of, among other things, an increased magnetic ripple. It should be noted that in both equilibria considered here, the tokamak-like ripple caused by finite coil number rather than plasma shaping is excluded from the calculation. It has been shown when considering a W7-X-like design alone that a careful consideration of the exact coil currents can make an important contribution to the final total energetic particle loss fraction ${ }^{34}$.

In figures $4 \mathrm{a}$ and $4 \mathrm{~b}$, loss fraction of alpha particles is plotted for a given initial distribution. Each line represents particles born at a given surface, labelled in terms of the square root of the normalised flux, $\rho=\sqrt{s} .4096$ particles are born at each surface. All particles are born with the D-T fusion alpha particle energy, $3.52 \mathrm{MeV}$, and a uniform distribution in pitch angle. They are followed

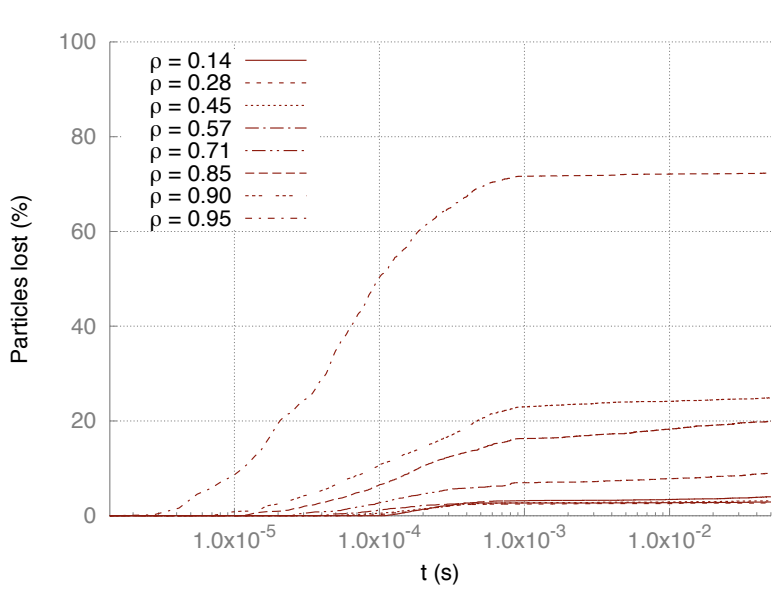

(a) Reactor-scale quasi-isodynamic stellarator.

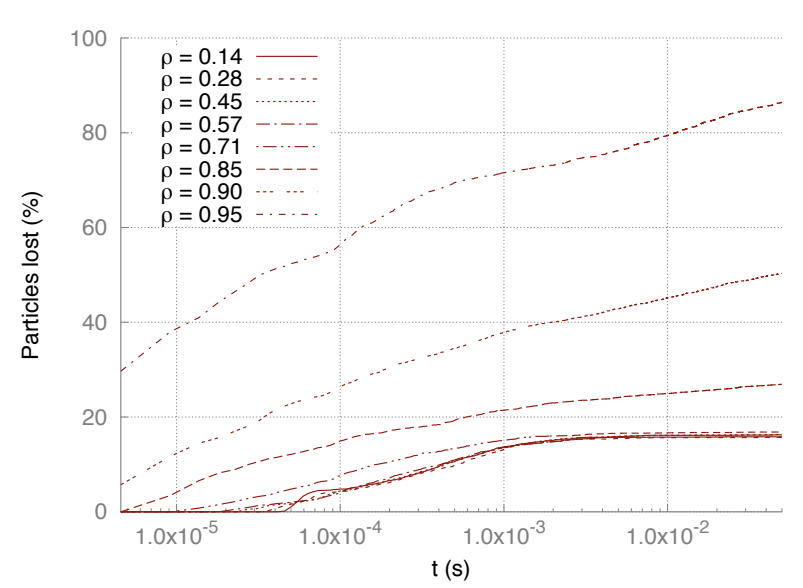

(b) The quasi-axisymmetric stellarator reactor ARIES-CS.

FIG. 4: Energetic particle loss fraction over time for alpha particles launched at different flux surfaces.

until $t=0.05 \mathrm{~s}$, or until they exit through the last closed flux surface. When all particles on initially unconfined orbits or orbits that pass frequently from locally trapped to passing regions, the loss fraction can reach a steady state. Otherwise, the simulations are terminated on a timescale comparable to the alpha particle thermalisation time.

The W7-X-like quasi-isodynamic design exhibits better collisionless alpha particle confinement than the quasiaxisymmetric design for particles born on the inner flux surfaces where most fusion reactions will take place. As one moves toward the edge of the device, confinement in the quasi-isodynamic machine worsens in relative terms and losses are greater for alpha particles born on the outermost flux surfaces. Sensitivity studies show that 


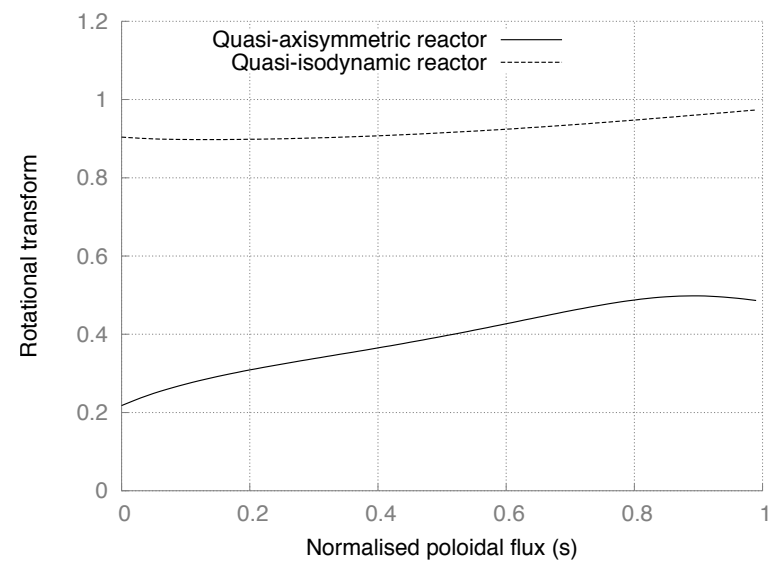

FIG. 5: The rotational transform profiles of the two reactors compared.

the pressure profile chosen for the equilibrium calculation may have a significant impact on these results for alphas born near the edge, and losses in this region for the quasiaxisymmetric reactor may be overstated. However, as few alpha particles are born or remain non-thermalised in this region, this effect should not be important.

In figures $6 \mathrm{a}$ and $6 \mathrm{~b}$, we plot the fraction of particles lost at $t=1 \times 10^{-5} \mathrm{~s}, t=1 \times 10^{-4} \mathrm{~s}$ and $t=1 \times 10^{-2} \mathrm{~s}$ at each initial flux surface for both the quasi-axisymmetric and the quasi-isodynamic devices. The prompt losses correspond to initially unconfined orbits, while the losses at a later time also include stochastic losses. Losses at timescales comparable to the device size over the thermal velocity of the energetic particles are likely to be dominated by prompt losses, while losses on a longer timescale are likely to be dominated by stochastic losses. Here, this characteristic timescale is $\sim 1 \times 10^{-6} \mathrm{~s}$. The evolving loss fraction for particles launched at different positions indicates the relative significance of the two mechanisms in each device. Stochastic losses are particularly severe in the quasi-axisymmetric design. They are the largest source of loss of particles born on the innermost flux surfaces where most fusion alpha particles will be generated.

In figures $7 \mathrm{a}$ and $7 \mathrm{~b}$, the loss fraction at $t=0.05 \mathrm{~s}$ is plotted for particles born with a given pitch angle at a given surface. Perhaps unsurprisingly, initially deeply trapped particles are more frequently lost than initially passing particles. The distribution of lost alphas is significantly different when comparing the two devices, however. In the quasi-axisymmetric design, almost all alphas born with a pitch angle greater than 50 degrees are lost regardless of the flux surface on which they are born. In the quasi-isodynamic design, by contrast, the distribution of loss with pitch angle is much more constant and there is no region in pitch angle where almost all fusion alpha particles are lost regardless of birth surface.

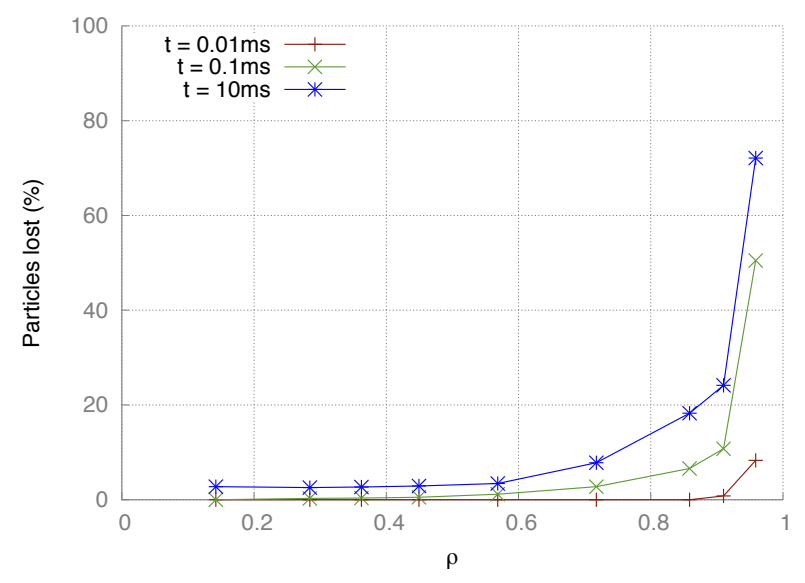

(a) Quasi-isodynamic stellarator.

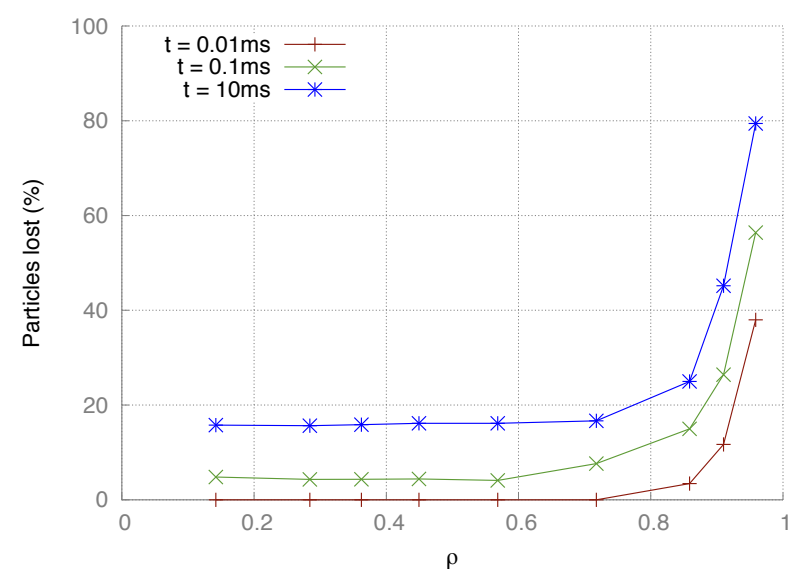

(b) ARIES-CS.

FIG. 6: Prompt and slow losses in stellarator reactors. Prompt losses are principally due to initially unconfined orbits, while slow losses are due to stochastic diffusion.

\section{CONCLUSION}

The whole volume, total-f gyrokinetic code XGC is being extended for stellarator geometries. Equilibrium magnetic field data has been generated for multiple representative stellarators and used by XGC. At this stage of development, the code has been verified against existing 3D codes, such as BEAMS3D ${ }^{31}$ and EUTERPE ${ }^{7}$, for orbit tracing. XGC has then been applied to calculate collisionless alpha particle confinement for possible stellarator reactors.

The quasi-axisymmetric design ARIES-CS has been compared with a Wendelstein 7-X-like quasi-isodynamic reactor design. In comparing the two proposed de- 

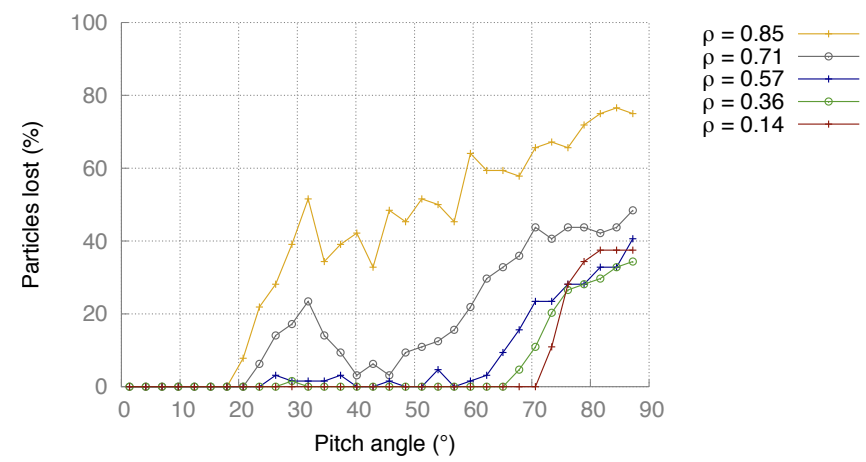

(a) Quasi-isodynamic stellarator.

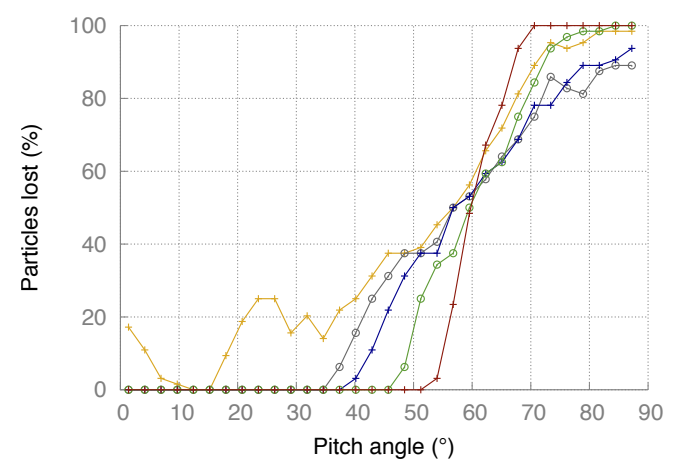

$\rho=0.85$ $\rho=0.71$ $\rho=0.57$
$\rho=0.36$ $\rho=0.36$

(b) ARIES-CS.

FIG. 7: Alpha particle losses by birth pitch angle in stellarator reactors.

signs considered here, it has been shown that the quasiisodynamic design has generally superior collisionless alpha particle confinement properties. This is driven by a reduced fraction of both prompt and slow stochastic losses for alpha particles born in the inner part of the volume where density and temperature, and therefore fusion cross section, will peak. It should be noted that future work in optimising stellarators for alpha particle confinement may well result in both quasi-isodynamic and quasi-axisymmetric designs with significantly better confinement, and this result should not be taken as representative for all possible designs in these broad classes.

In future work, XGC will be further extended to include a Poisson solver, initially assuming that perturbations are extended along field lines which are perpen- dicular to unstructured poloidal meshes, and eventually extended to full 3D. This will permit simulations of bulk plasma phenomena such as ion-scale turbulence in stellarators. At the same time, work is on-going extending the equilibrium magnetic field and solver to encompass the edge region. Equilibrium magnetic field calculations will also be extended to include non-ideal effects such as magnetic islands and stochastic regions, calculated by a code such as SPEC $^{35,36}$. Collisional effects will also be considered $^{37}$.

\section{ACKNOWLEDGMENTS}

The authors would like to thank J. Riemann, M. Borchardt and J. Geiger for their assistance with the EUTERPE and VMEC codes. We are thankful for the work of S. Hirshman in developing and maintaining the VMEC code. We thank P. Helander for carefully reviewing this manuscript, but any remaining errors are our own. We gratefully acknowledge the continuing support of Michael Zarnstorff for this work. This manuscript has been authored by Princeton University under Contract Number DE-AC02-09CH11466. The United States Government retains and the publisher, by accepting the article for publication, acknowledges that the United States Government retains a non-exclusive, paid-up, irrevocable, world-wide license to publish or reproduce the published form of this manuscript, or allow others to do so, for United States Government purposes.

${ }^{1}$ L. Spitzer, The Physics of Fluids 1, 253 (1958).

${ }^{2}$ P. Xanthopoulos, H. E. Mynick, P. Helander, Y. Turkin, G. G. Plunk, F. Jenko, T. Görler, D. Told, T. Bird, and J. H. E. Proll, Phys. Rev. Lett. 113, 155001 (2014).

${ }^{3}$ H. Sugama and W. Horton, Phys. Plasmas 2, 2989 (1995).

${ }^{4}$ H. Sugama, T. Watanabe, M. Nunami, S. Satake, S. Matsuoka, and K. Tanaka, Plasma Fusion Res. 7, 2403094 (2012).

${ }^{5}$ M. Oberparleiter, F. Jenko, D. Told, H. Doerk, and T. Görler, Phys. Plasmas 23, 042509 (2016).

${ }^{6}$ P. Helander, C. D. Beidler, T. M. Bird, M. Drevlak, Y. Feng, R. Hatzky, F. Jenko, R. Kleiber, J. H. E. Proll, Y. Turkin, and P. Xanthopoulos, Plasma Phys. Control. Fusion 54, 124009 (2012).

${ }^{7}$ V. Kornilov, R. Kleiber, R. Hatzky, L. Villard, and G. Jost, Phys. Plasmas 11, 3196 (2004).

${ }^{8}$ J. M. García-Regaña, R. Kleiber, C. D. Beidler, Y. Turkin, H. Maaßberg, and P. Helander, Plasma Phys. Control. Fusion 55, 074008 (2013).

${ }^{9}$ M. A. Pedrosa, J. A. Alonso, J. M. García-Regaña, C. Hidalgo, J. L. Velasco, I. Calvo, R. Kleiber, C. Silva, and P. Helander, Nucl. Fusion 55, 052001 (2015).

${ }^{10}$ J. M. García-Regaña, C. D. Beidler, R. Kleiber, P. Helander, A. Mollén, J. A. Alonso, M. Landreman, H. Maaßberg, H. M. Smith, Y. Turkin, and J. L. Velasco, Nucl. Fusion 57, 056004 (2017).

${ }^{11}$ P. Helander, T. Bird, R. Kleiber, G. G. Plunk, J. H. E. Proll, J. Riemann, and P. Xanthopoulos, Nucl. Fusion 55, 053030 (2015).

${ }^{12}$ A. Mishchenko, M. Borchardt, M. Cole, R. Hatzky, T. Fehér, R. Kleiber, A. Könies, and A. Zocco, Nucl. Fusion 55, 053006 (2015).

${ }^{13}$ J. Riemann, R. Kleiber, and M. Borchardt, Plasma Phys. Control. Fusion 58, 074001 (2016). 
${ }^{14}$ A. Mishchenko, A. Könies, T. Fehér, R. Kleiber, M. Borchardt, J. Riemann, R. Hatzky, J. Geiger, and Y. Turkin, Nucl. Fusion 54, 104003 (2014).

${ }^{15}$ D. A. Spong, I. Holod, Y. Todo, and M. Osakabe, Nucl. Fusion 57, 086018 (2017)

${ }^{16} \mathrm{~S}$. Matsuoka, Y. Idomura, and S. Satake, Phys. Plasmas 25, 022510 (2018).

${ }^{17}$ S. H. Ku, C. S. Chang, and P. Diamond, Nucl. Fusion 49, 115021 (2009).

${ }^{18}$ S. H. Ku, R. Hager, C. S. Chang, J. Kwon, and S. Parker, J. Comp. Physics 315, 467 (2016).

${ }^{19}$ S. Ku, C. S. Chang, R. Hager, R. M. Churchill, G. R. Tynan, I. Cziegler, M. Greenwald, J. Hughes, S. E. Parker, M. F. Adams, E. D'Azevedo, and P. Worley, Phys. Plasmas 25, 056107 (2018).

${ }^{20}$ R. M. Churchill, C. S. Chang, S. Ku, and J. Dominski, Plasma Phys. Control. Fusion 59, 105014 (2017).

${ }^{21}$ C. Chang, S. Ku, G. Tynan, R. Hager, R. Churchill, I. Cziegler, M. Greenwald, A. Hubbard, and J. Hughes, Phys. Rev. Lett. 118, 175001 (2017).

${ }^{22}$ S. P. Hirshman and J. C. Whitson, Phys. Fluids 26, 355368 (1983).

${ }^{23}$ W. Suttrop, T. Eich, J. C. Fuchs, S. Günter, A. Janzer, A. Herrmann, A. Kallenbach, P. T. Lang, T. Lunt, M. Maraschek, R. M. McDermott, A. Mlynek, T. Pütterich, M. Rott, T. Vierle, E. Wolfrum, Q. Yu, I. Zammuto, and H. Zohm, Phys. Rev. Lett 106, 225004 (2011).

${ }^{24}$ O. Schmitz, K. Ida, M. Kobayashi, A. Bader, S. Brezinsek, T. E. Evans, H. Funaba, M. Goto, O. Mitarai, and T. Morisaki, Nucl. Fusion 56 (2016)

${ }^{25}$ P. Helander, Rep. Prog. Phys. 77, 087001 (2014).

${ }^{26}$ W. Lotz, P. Merkel, J. Nuhrenberg, and E. Strumberger, Plasma Phys. Control. Fusion 34, 1037 (1992)

${ }^{27}$ C. D. Beidler, Y. I. Kolesnichenko, V. S. Marchenko, I. N. Sidorenko, and H. Wobig, Phys. Plasmas 8, 2731 (2001).
${ }^{28}$ C. Beidler, K. Allmaier, M. Isaev, S. Kasilov, W. Kernbichler, G. Leitold, H. Maaßberg, D. Mikkelsen, S. Murakami, M. Schmidt, D. Spong, V. Tribaldos, and A. Wakasa, Nucl. Fusion 51 (2011).

${ }^{29}$ T. S. Pedersen, T. Andreeva, H.-S. Bosch, S. Bozhenkov, F. Effenberg, M. Endler, Y. Feng, D. Gates, J. Geiger, D. Hartmann, H. Hölbe, M. Jakubowski, R. König, H. Laqua, S. Lazerson, M. Otte, M. Preynas, O. Schmitz, T. Stange, Y. Turkin, and the W7-X Team, Nucl. Fusion 55, 126001 (2015).

${ }^{30}$ J. Geiger, C. D. Beidler, Y. Feng, H. Maaßberg, N. B. Marushchenko, and Y. Turkin, Plasma Phys. Control. Fusion 57 (2015).

${ }^{31}$ M. McMillan and S. A. Lazerson, Plasma Phys. Control. Fusion 56, 095019 (2014).

${ }^{32}$ E. Strumberger, Nucl. Fusion 40 (2000).

${ }^{33}$ F. Najmabadi, A. R. Raffray, S. I. Abdel-Khalik, L. Bromberg, L. Crosatti, L. El-Guebaly, P. R. Garabedian, A. A. Grossman, D. Henderson, A. Ibrahim, T. Ihli, T. B. Kaiser, B. Kiedrowski, L. P. Ku, J. F. Lyon, R. Maingi, S. Malang, C. Martin, T. K. Mau, B. Merrill, R. L. Moore, R. J. P. Jr., D. A. Petti, D. L. Sadowski, M. Sawan, J. H. Schultz, R. Slaybaugh, K. T. Slattery, G. Sviatoslavsky, A. Turnbull, L. M. Waganer, X. R. Wang, J. B. Weathers, P. Wilson, J. C. W. III, M. Yoda, and M. Zarnstorff, Fusion Sci Technol. 54, 655 (2008).

${ }^{34}$ M. Drevlak, J. Geiger, P. Helander, and Y. Turkin, Nucl. Fusion 54, $073002(2014)$.

${ }^{35}$ S. R. Hudson, R. L. Dewar, G. Dennis, M. J. Hole, M. McGann, G. von Nessi, and S. Lazerson, Phys. Plasmas 19, 112502 (2012).

${ }^{36}$ J. Loizu, S. R. Hudson, and C. Nührenberg, Phys. Plasmas 23, 112505 (2016).

${ }^{37}$ R. Hager, E. S. Yoon, S. Ku, E. F. D'Azevedo, P. H. Worley, and C. S. Chang, J. Comp. Physics 315, 644 (2016). 